\title{
Application of optical heterodyning methods in microwave transducers
}

\author{
Vladimir Biryukov ${ }^{1}$, Vladimir Grachev ${ }^{1}$, Sergey Kapustin ${ }^{1, *}$, Kirill Malyshev ${ }^{1}$, \\ Yuliya Perevezentseva ${ }^{1}$, and Alexey Raevsky ${ }^{1}$ \\ ${ }^{1}$ Nizhny Novgorod State Technical University n.a. R.E. Alekseev \\ Minin St., 24, Nizhny Novgorod, 603950, Russia
}

\begin{abstract}
An optical signal mixes up with the reference radiation of heterodyne on a sensitive photodetector site. A beat signal with wavelength separation frequency of heterodyne and information light signal is generated at the output of the photodetector. This beat frequency emittedby the detector must be in radio wave range; this means that the received signal can be processed electronically.
\end{abstract}

\section{Introduction}

Microwave signal generators are used as a part of various electronic equipment as well as telecoms, radio aids and metrology tools. Improving their basic characteristics, such as a widely-turned range, the level of jitter, frequency stability and output power, is an urgent task. Therefore, the design of generator-based photonic technologies is an outlook study. There are two approaches to receive radio signals through photonic methodology nowadays: self-generation signals [1-2] and optical heterodyning [3-7].

\section{Self-generation signals}

An optoelectronic generator (OEG) is a sort of self-generator. It almost overcomes all limitations of generators using traditional building techniques. First of all, the OEG eliminates a compromise between a widely-turned range and the level of jitter However, one of the main characteristics of the OEG, affecting noise and spectral properties of the generator, is a signal delay time in the feedback loop. This period is determined by the length of the fiber optic path used in the OEG.

A structure flowchart of the OEG is shown in Figure 1.

This flowchart includes optical and radio nodes. An optical node consists of: a semiconductor laser module (SCLM); a radiation intensity modulator (RIM); a fiber optic path (FOP); and a photodiode module (PDM). A radio node consists of a preamplifier (PA), a pass band filter (PBF), an amplifier and a power splitter (PS).

*Corresponding author: Kapustin_1994@mail.ru 


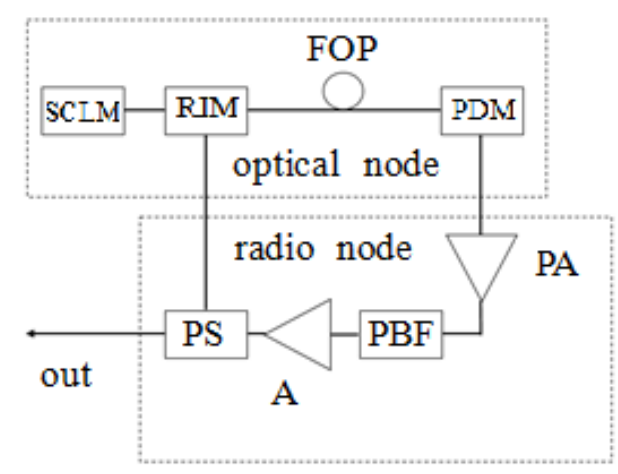

Fig. 1. Structure flowchart of the OEG.

For the stationary operation of an optoelectronic generator, continuous radiation of the SCLM arrives at the input of the RIM. After that, the optical radiation, modulated by a microwave signal, passes through the fiber-optic path and comes on the input of the PDM where the signal is demodulated. The demodulated microwave signal is pre-amplified by an electric amplifier.

High-Q PBF shows a generation frequency of the OEG and kills microwave oscillation at other frequencies. Having amplified, the microwave signal comes to the PS which sends a certain portion of the signal to the output of the OEG and the other one to the control input of the RIM. As a result, a positive feedback loop is closed.

The self-excitation process of the OEG is caused by fluctuations in the elements of its scheme; for example, laser intensity fluctuations, shot noise at the output of photodiode, thermal noise in the electronic elements. Because of positive feedback, vibrational amplitude increases exponentially. However, with more oscillation in amplitude, there is more nonlinearity of the feedback loop. As a result, the OEG total gain decreases and the generator goes into a stationary mode.

Static operation of the OEG as with any feedback generator is achieved by amplification in the preamplifier and the amplifier, which compensate electro-optical conversion losses in the OEG (amplitude balance), as well as by matching the signal delay time in the feedback loop to the phase progression which equals to $2 \pi \mathrm{k}$, where $\mathrm{k}=1,2, \ldots$ (phase balance).

\section{Optical heterodyning}

The method of optical heterodyning is the addition of two optical waves with different frequencies on a sensitive photodetector site. A qualitative structural diagram is shown in Figure 2.

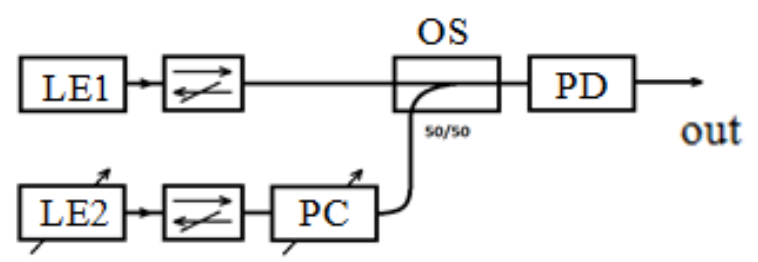

Fig. 2. Structure flowchart of optical heterodyning. 
A beat signal with wavelength separation frequency of fixed and tunable laser emitters is generated at the output of the photodetector. This beat frequency, emitted by the detector, must be in the radio or microwave spectrum which can be processed electronically. This method has high sensitivity which is determined by an amplification effect. This effect is that desired signal range at the output of the heterodyne photodetector is proportional to the product of the amplitudes of two signals.

After hitting the photodetector site, wave vectors must be parallel to each other while the vector E must range within the same plane.

Parallelism of vectors is achieved by use of an optical fiber while E-vibrations is achieved by use of a polarization controller in one of the lines. Beat signals with total and difference frequencies are generated. However, the signal with total frequency is excluded from consideration because it does not fall into the photodiode passband.

The beat frequency can reach the terahertz range; only the photodetector bandwidth can be a limitation.

However, if two non-synchronized laser diodes are used, a received radio-frequency signal will have a high level of phase noise because the phases of two waves are not correlated.

There are several methods of phase locking for two optical waves. These methods include techniques of frequency-lock synchronization, phase-lock control and combined approach.

A special case of heterodyning (homodyne detection as the easiest way to implement the method) is considered to analyze the laser spectrum.

A laboratory setup of a receive path with optical homodyning was developed and assembled. This scheme is shown in Figure 3.

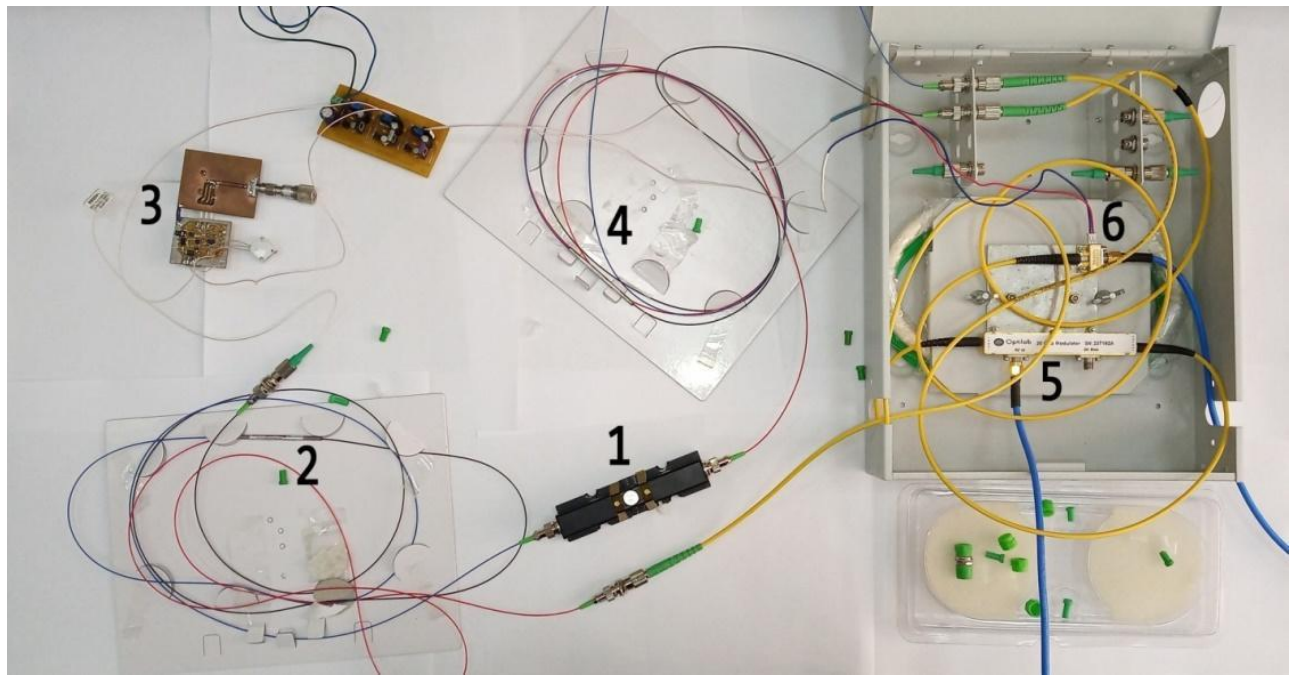

Fig. 3. Scheme with optical homodyning:

1. Polarization controller

2. Optical combiner

3. Laser

4. Optical splitter

5. Radiation intensity modulator

6. Photodiode

A laser signal enters the splitter. One splitter line goes to the polarization controller, which is configured for polarization with maximum capacity, the other one goes to the radiation intensity modulator where radiation is modulated by a $1 \mathrm{GHz}$ microwave signal. 
Two signals from the polarization controller and from the modulator go to the optical combiner and merge together on the photodiode. The signal from the photodiode is observed on a spectrum analyzer (Figure 4).

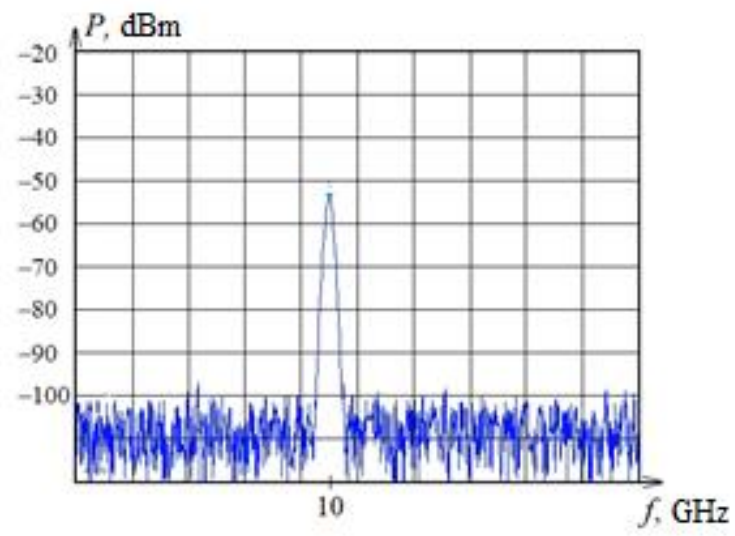

Fig. 4. The signal on the spectrum analyzer during homodyne detection.

A - $54 \mathrm{dBm}$ signal is observed. After making a break in the splitter line, the direct detection of optical signal arises. The results are shown in Figure 5.

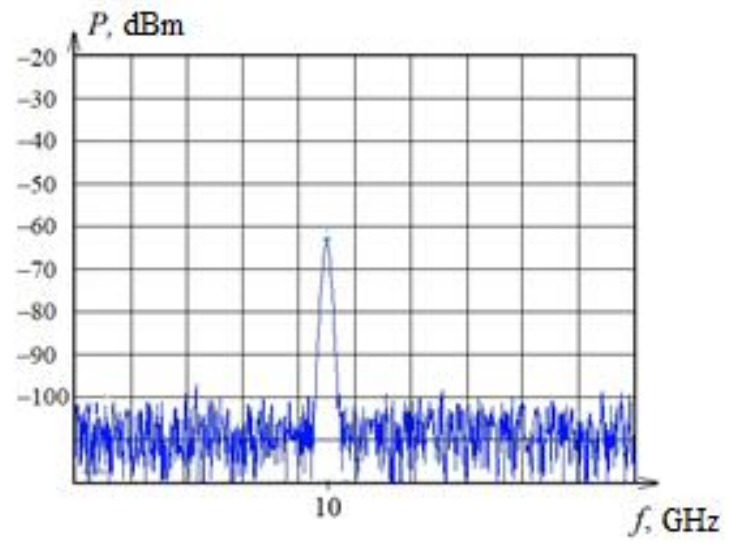

Fig. 5. The signal on the spectrum analyzer during direct detection.

The figure shows that the homodyne method gives a signal power advantage in comparison with direct detection at $10 \mathrm{~dB}$.

\section{Summary}

By use of optical heterogenerous, spread spectrum microwave signals can be formed. To form microwave signals with ultra-low phase noise, it is necessary to synchronize laser sources. Modern element base of radio photonics allows to create the phase synchronization systems with simple methods. Optical heterodination systems in comparison with quadratic detection additionally allow to amplify the microwave signals by changing the heterodyne power, i.e. optical heterodination systems increase the sensitivity of a broadband communication system, which was demonstrated by the example of the homodyne method.

The report was prepared on the basis of a study carried out as part of the project of the Russian Science Foundation No. 17-19-01628. 


\section{References}

1. 1 V.V. Biryukov, V.A. Grachev, S.A. Kapustin, T.S. Lukoyanova, S.G. Lobin, A.S. Raevskii, Proceedings of SPIE, Signature analysis of microwave signal generator with a fiber optic delay line in a feedback loop, Vol. 9807, 980712 (2016)

2. V.V. Biryukov, V.A. Grachev, S.A. Kapustin, S.G. Lobin, A.S. Raevskii, Proceedings of SPIE, Investigation of the characteristics of fiber optic delay lines with different types of optical emission intensity modulation, Vol. 10342, 103420K (2017)

3. Y. Wu, X. B. Xie, J. H. Hodiak, IEEE photonics technology letters, Multioctave high dynamic range up-conversion optical-heterodyned microwave photonic link, Vol. 16, No. 10, pp. 23322334 (2004)

4. J. Zacharias, An. Krishnan, J. Joy, S. Elizabeth, V. Narayanan, International Conference on Trends in Electronics and Informatics, Full duplex millimeter-wave radio-over-fiber system using optical heterodyning and self-homodyning, pp. 378-381 (2017)

5. M. Chen, J. Yu, X. Xiao, IEEE Photonics Journal, Real-time Q-band OFDM-RoF systems with optical heterodyning and envelope detection for downlink transmission, Vol. 9, No 2, 7902007 (2017)

6. V.V. Kulagin, V.V. Valuev, V. A. Cherepenin, International Conference on Microwave and Photonics, Optical heterodyning in microwave photonic receiver for radar applications, 15801385 (2016)

7. F. Paresys, T. Shao, G. Maury, Y. Le Guennec, B. Cabon, Opt. Commun. Netw., Bidirectional millimeter-wave radio-over-fiber system based on photodiode mixing and optical heterodyning, Vol. 5, No. 1, pp. 74-80 (2013) 